\title{
Determinación de linfocitos T CD4, CD8 y T $\gamma \delta$ en la infección experimental con Brucella ovis
}

\author{
Determination of T CD4, CD8 and T $\gamma \delta$ lymphocytes in experimental \\ infection with Brucella ovis
}

\author{
Víctor Manuel Sánchez-Parra ${ }^{1,2}$, Edgardo Soriano-Vargas ${ }^{1}$, Ruy Ortiz-Rodríguez ${ }^{2}$, \\ Esvieta Tenorio Borroto ${ }^{1}$, Valente Velázquez-Ordoñez ${ }^{1}$, Martín Talavera-Rojas ${ }^{1}$, \\ Jorge Acosta-Dibarrat ${ }^{1,3}$
}

\section{Resumen}

El objetivo de este estudio fue determinar la distribución sanguínea de linfocitos T CD4, CD8, LT $\gamma \delta$ (WC1) en carneros infectados experimentalmente con Brucella ovis. Se utilizaron 18 carneros de 1 a 4 años y libres de $B$. ovis distribuidos en tres grupos: Control $(n=6)$; Inoculado en las mucosas ocular y prepucial $(n=6)$; Inoculados vía endovenosa $(n=6)$. Se realizó el seguimiento serológico desde el día de la inoculación hasta el $189 \mathrm{dPI}$ (día pos-inoculación). Se inmunotipificaron las poblaciones de linfocitos CD4 y CD8 a los 120, 150 y 189 y de WC1 (linfocitos T $\gamma \delta$ ) a los 120 dPI por citometría de flujo. Los carneros, a partir del tercer dPI comenzaron a seroconvertir; a los 21 y $28 \mathrm{dPI}$ todos los animales de los grupos inoculados por vía endovenosa y mucosa, respectivamente, resultaron positivos; asimismo, el 50\% de los animales de los grupos desafiados se presentaron como positivos o sospechosos a la prueba de ELISA a los $189 \mathrm{dPI}$. Se encontraron diferencias en las Medias de Intensidad de Fluorescencia (MIF) de los linfocitos CD8 entre el grupo control (1027.4) y el grupo inoculado por vía endovenosa (499.6) a los $120 \mathrm{dPI}(\mathrm{p}<0.05)$ y en las MIF de los linfocitos CD4 entre grupos a los $189 \mathrm{dPI}$ $(\mathrm{p}<0.05)$. Las poblaciones linfocitarias $\mathrm{T} \gamma \delta(\mathrm{WC} 1)$ presentaron diferencias en MIF entre el grupo control y los grupos inoculados a los $120 \mathrm{dPI}(\mathrm{p}<0.05)$. Los resultados indican que $B$. ovis puede modular la respuesta inmune del hospedero, que los linfocitos CD4 y CD8 son importantes para la defensa del hospedero ante esta infección, y que las poblaciones de CD4 como de CD8 pueden fluctuar durante el periodo de infección por B. ovis. Así mismo, la participación de los linfocitos $\mathrm{T} \gamma \delta$ podría ser un factor importante en el control de la infección causada por B. ovis.

Palabras clave: carneros; epididimitis ovina; Brucella ovis; linfocitos T; ELISA; citometría de flujo

\footnotetext{
${ }^{1}$ Centro de Investigación y Estudios Avanzados en Salud Animal, Facultad de Medicina Veterinaria y Zootecnia, Universidad Autónoma del Estado de México, México

${ }^{2}$ Facultad de Medicina Veterinaria y Zootecnia, Universidad Michoacana de San Nicolás de Hidalgo, Michoacán, México

${ }^{3}$ E-mail:jpacostad@uaemex.mx
}

Recibido: 16 de octubre de 2018

Aceptado para publicación: 3 de julio de 2019 
The objective of this study was to determine the blood distribution of CD4, CD8, and $\mathrm{T} \gamma \delta$ lymphocytes (WC1) in rams experimentally infected with Brucella ovis. Eighteen rams from 1 to 4 years old and free from $B$. ovis were distributed in three groups: Control $(n=6)$; Inoculated in the ocular and preputial mucosa $(n=6)$; Inoculated intravenously $(n=6)$. Serological follow-up was carried out from the day of inoculation until $189 \mathrm{dPI}$ (post-inoculation day). CD4 and CD8 lymphocyte populations were immunotyped at 120, 150 and 189 and WC1 (T $\gamma \delta$ lymphocytes) at $120 \mathrm{dPI}$ by flow cytometry. The rams, from the third dIP began to seroconvert; at 21 and $28 \mathrm{dPI}$ all animals of the inoculated intravenously and in the mucosa groups, respectively, were positive; likewise, $50 \%$ of the animals of the challenged groups were presented as positive or suspect to the ELISA test at $189 \mathrm{dPI}$. Differences were found in the Mean Fluorescence Intensity (MFI) of the CD8 lymphocytes between the control group (1027.4) and the group inoculated intravenously (499.6) at $120 \mathrm{dPI}(\mathrm{p}<0.05)$ and in the MFI of the lymphocytes CD4 between groups at $189 \mathrm{dPI}(\mathrm{p}<0.05)$. The $\mathrm{T} \gamma \delta$ lymphocyte populations (WC1) presented differences in MFI between the control group and the groups inoculated at $120 \mathrm{dPI}(\mathrm{p}<0.05)$. The results indicated that $B$. ovis can modulate host immune response, that CD4 and CD8 lymphocytes are important for host defence against this infection, and that populations of CD4 and CD8 can fluctuate during the period of $B$. ovis infection. Besides, the participation of $\mathrm{T} \gamma \delta$ lymphocytes could be an important factor in the control of the infection caused by $B$. ovis.

Key words: ovine epididymitis; Brucella ovis; AGID; ELISA; flow cytometry

\section{INTRODUCCIÓN}

La epididimitis causada por Brucella ovis (B. ovis) es una enfermedad contagiosa de transmisión venérea que causa falla reproductiva (Moustacas et al., 2013; Costa et al., 2016), y se caracteriza por producir epididimitis, subfertilidad e infertilidad en carneros (Ovis aries) (Galindo et al., 2009b; Ridler et al., 2014). En los carneros, las lesiones crónicas causadas por $B$. ovis se restringen al aparato genital, específicamente al epidídimo y eventualmente testículos y glándulas asociadas al aparato reproductor (Xavier et al., 2010).

El carácter intracelular facultativo de Brucella condiciona la respuesta inmune del hospedador. Se trata de una respuesta inmunitaria mixta, de tipo humoral y celular. La respuesta inmunitaria humoral a $B$. ovis se encuentra dirigida principalmente contra el lipopolisacárido rugoso (LPS-R) y las proteínas de membrana externa (PME) (Jaques et al., 1992; Bowden et al., 1995). Por otro lado, estudios experimentales demostraron que la inmunidad humoral es tan importante como la inmunidad celular en la defensa contra la brucelosis, aunque se desconoce las células implicadas en la respuesta (Estein $e t$ al., 2004). El predominio de los isotipos $\operatorname{IgG}_{2 \mathrm{a}}$ e $\mathrm{IgG}_{3}$ en ratón permitiría inferir que $B$. ovis induce la población de linfocitos T colaboradores del tipo 1 (LTh1) productores de interferón $\gamma$ (Galindo et al., 2009a; Praud et al., 2012).

Las células T CD4 se describen generalmente como células «auxiliares». Tienen un papel importante en el control y regulación del sistema inmune colaborando en la activación de las células B y en la generación de células T CD8 citotóxicas (Allen y Maizels, 1997; Cunnusamy et al., 2010). La heterogeneidad de las células $\mathrm{T}$ se describió 
por primera vez hace dos décadas con la descripción de las células TH1 y TH2 (Issuree et al., 2017; Cobbold et al., 2018). Las células TH1 están involucradas en la defensa del huésped contra patógenos intracelulares y células tumorales produciendo altos niveles de IFN- $\gamma$, TNF- $\alpha$ e IL-2. Las células TH2 son responsables de coordinar la inmunidad humoral e inflamación eosinofílica y están involucradas en la defensa del huésped contra parásitos extracelulares, secretando IL4, IL-5, IL-10 y IL-13. Nuevos subconjuntos (TH9, TH22, TH17, THFH, NKT, células Treg) han sido descritos confirmando la heterogeneidad de la familia de células T CD4 (Mucida y Cheroutre, 2010; Hawkins et al., 2013). Varios estudios en ratones y humanos han reportado un potencial citotóxico de las células T CD4 (van der Veken et al., 2005).

Aunque la inmunidad innata es capaz de controlar las brucellas, la inmunidad específica es necesaria para montar una fuerte respuesta inmune en etapas tardías de la infección (Baldwin y Goenka, 2006). Se ha descrito ampliamente que la respuesta $\mathrm{TH} 1$ mediadas por IFN- $\gamma$ son esenciales para el aclaramiento del patógeno. De hecho, IFN- $\gamma$ producido por células T CD4 y las células T $\gamma \delta$ han demostrado activar las propiedades bactericidas en macrófagos para dificultar la supervivencia intrahuésped de la Brucella, mientras que IFN- $\gamma$ producido por células $\mathrm{T}$ CD8 y la inmunidad humoral relacionada con las células B tienen roles modestos contra la infección por Brucella en ratones (Skyberg et al., 2011; Vitry et al., 2012). En otros estudios se demostró que los isotipos de anticuerpos tipo $\mathrm{TH} 1$, tales como $\mathrm{IgG}_{2 \mathrm{a}} \mathrm{IgG}_{3}$ opsonizan la Brucella para facilitar la fagocitosis y la liberación bacteriana en compartimentos endocíticos degradativos (Goenka et al., 2011). En los seres humanos, las células T CD4 productoras de IFN- $\gamma$, las células T CD8 y las células $\mathrm{T} \gamma \delta$ han sido implicadas en el control de la brucelosis (Bessoles et al., 2011; Cannella et al., 2012).
Con respecto a la distribución de los linfocitos $\mathrm{T} \gamma \delta$, se considera que su proporción con respecto a otros linfocitos es reducida en los órganos linfoides; sin embargo, aumentan su proporción en la piel y superficies mucosas, lo que sugiere que tienen un papel importante en la vigilancia y repuesta inmediata a los patógenos invasores (Dudal et al., 2006; Plattner y Hostetter, 2011; Acosta-Dibarrat et al., 2014, 2016). Los linfocitos T $\gamma \delta$ representan entre 1 y $10 \%$ de los linfocitos $\mathrm{T}$ circulantes en humanos adultos y ratones. En el caso de los ovinos es del $17 \%$ y en bovinos adultos entre 10 y $25 \%$, aunque pueden llegar al $40 \%$ en terneros jóvenes (Plattner y Hostetter, 2011; Ni et al., 2012). Por lo tanto, el objetivo de este estudio fue caracterizar la distribución en sangre de linfocitos T CD4, CD8, LT $\gamma \delta$ (WC1) en carneros con infección experimental crónica con Brucella ovis por vía endovenosa y por mucosa.

\section{Materiales y Métodos}

El estudio se realizó en el Centro de Investigación y Estudios Avanzados en Salud Animal de la Universidad Autónoma del estado de México (UAEM) $\left(40^{\circ} 24^{\prime} 59,87^{\prime \prime} \mathrm{N}\right)$, entre los meses de marzo y noviembre de 2012. El estudio fue aprobado por el Comité de Bioética de la Facultad de Medicina Veterinaria y Zootecnia de la UAEM.

\section{Animales}

Se emplearon 18 carneros de las razas Columbia, Romanov y Pelibuey, con edad entre 1 y 4 años. Los carneros procedían de un establecimiento libre de B. ovis. Se constató que los animales no presentaran signos de epididimitis mediante el examen clínico. Además, se les tomó muestras para el análisis serológico de immunodifusión en gel de agar(IGDA) y ELISA, así como cultivo bacteriológico del semen y del lavado prepucial, 
dando resultados negativos en todos los casos. Los animales fueron alimentados ad libitum con heno de avena (Avena sativa) y alimento comercial ( $14 \%$ de proteína cruda).

Los carneros se distribuyeron aleatoriamente en tres grupos $(n=6)$ : Grupo 1: control negativo, se le administró instilación conjuntival y prepucial de $0.5 \mathrm{ml}$ de solución salina al 0.9\% (SS); Grupo 2: inoculación en mucosas: instilación de $B$. ovis en la mucosa conjuntival y prepucial con $0.5 \mathrm{ml}$ de inóculo con una concentración de $3.9 \times 10^{9}$ UFC (unidades formadoras de colonias) en ambas mucosas; Grupo 3: vía intravenosa, $1 \mathrm{ml} \mathrm{del}$ inóculo en la vena yugular a la misma concentración. La instilación en conjuntiva se realizó en el ojo izquierdo, mientras que para la administración prepucial se colocó el inóculo o la SS usando una jeringa sin aguja y posterior masaje con la abertura prepucial cerrada.

\section{Inóculo de Desafío}

Se inocularon placas de agar sangre con la cepa de campo B. ovis 01Zac-INIFAP obtenida del epidídimo de un carnero con epididimitis en el estado de Zacatecas, México. Se incubaron a $37^{\circ} \mathrm{C}$ durante $48 \mathrm{~h}$, en una atmósfera con $10 \%$ de $\mathrm{CO}_{2}$. Se cosecharon con SS. Al inóculo se le realizó conteo de UFC (Miles et al., 1938) y se estandarizó a una concentración de $3.9 \times 10^{9} \mathrm{UFC} / \mathrm{ml}$.

\section{Muestreo}

Se colectaron muestras de sueros y de semen de todos los carneros para realizar pruebas serológicas de ELISA y detectar $B$. ovis por PCR durante seis meses (días 0,3 , $7,13,21,28,42,56,70,80,120,150$ y 189 ). Las muestras de sangre $(5 \mathrm{ml})$, se obtuvieron en tubos estériles (BD Franklin Lake NJ, USA), se separó el suero por centrifugación (Becton Dickinson Clay Adams, USA) y se conservaron a $-80{ }^{\circ} \mathrm{C}$ (Thermo Fisher Scientific/ Revco, ULT1786-3-A40, USA).
Se tomaron 4-5 ml de muestras de sangre a los carneros para realizar la fenotipificación de CD4 y CD8 a los 120, 150 y 189 días y de $\mathrm{WC1}$ (linfocitos $\mathrm{T} \gamma \delta$ ) a los 120 días del desafío. Las muestras se llevaron al laboratorio y se mantuvieron a $4{ }^{\circ} \mathrm{C}$ hasta su procesamiento dentro de las $12 \mathrm{~h}$ posteriores a la extracción utilizando citometría de flujo.

\section{Prueba de ELISA}

Se utilizó el kit de ELISA (CHEKIT Brucella ovis, IDEXX) para detección de anticuerpos de $B$. ovis en suero, siguiendo las recomendaciones del fabricante. El kit posee placas de 96 pozos sensibilizadas con el antígeno, sueros controles positivo y negativo, solución tampón para lavado y la dilución de los sueros (anticuerpo anti-IgG ovina conjugado con peroxidasa), sustrato y solución de paro de la reacción.

\section{Inmunofenotipificación de Poblaciones Linfocitarias}

Se obtuvieron $500 \mu 1$ de sangre periférica que se diluyeron en $500 \mu 1$ de PBS-BSA (1:1). La separación de los linfocitos se hizo empleando $3 \mathrm{ml}$ de Lymphoprep (Stemcell, Canadá) a $4{ }^{\circ} \mathrm{C}$. El anillo obtenido se centrifugó a $80 \mathrm{~g}$ durante 20 minutos. El tapón linfocitario fue suspendido en $2 \mathrm{ml}$ de PBS-BCA para su lavado. Las células leucocitarias fueron suspendidas en $1000 \mu 1$ de PBS-BSA a $4{ }^{\circ} \mathrm{C}$. Para el marcaje se tomó $150 \mu 1$ de la muestra y se adicionó $50 \mu 1$ de los anticuerpos monoclonales (CD4 Alexa Fluor, CD8 PE, WC1 FITC, Laboratorio Serotec) con reactividad para ovino (Navarro et al., 1996). Esto se repitió en cada uno de los muestreos de los carneros de los tres grupos.

\section{Análisis Estadístico}

El análisis de las poblaciones (medido en Medias de Intensidad de Fluorescencia MIF) de los linfocitos durante la fase experimental se realizó a través de la metodología de mediciones repetidas utilizando para el 


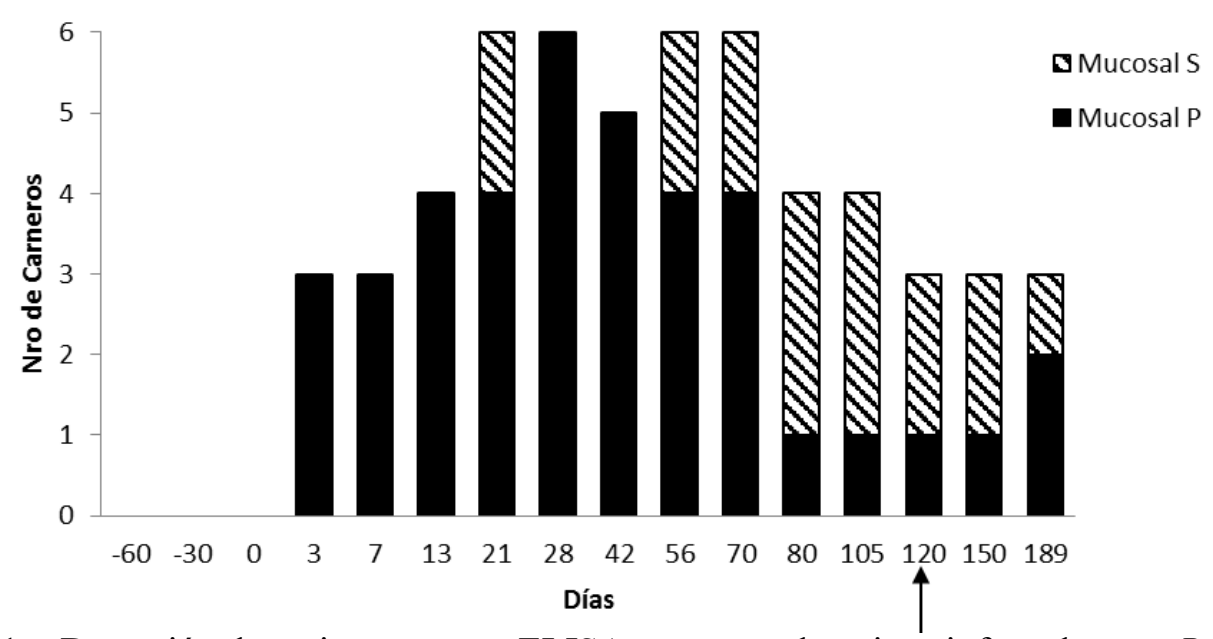

Figura 1. Detección de anticuerpos por ELISA en sueros de ovinos infectados con B. ovis en la mucosa conjuntival y prepucial con $0.5 \mathrm{ml}$ de inóculo con una concentración de 3.9x $10^{9}$ UFC. S: sospechoso; P: positivo. La flecha indica el momento en que se comenzó a realizar las lecturas de poblaciones linfocitarias (día 120 pos-infección)

modelo de efectos fijos y las diferencias entre grupos y tiempo se obtuvieron mediante la prueba de medias de mínimos cuadrados a un $\alpha=0.05$ (Littell et al., 1998). Se empleó el software InfoStat.

\section{Resultados}

\section{Serología}

Las Figuras 1 y 2 muestran los resultados de las pruebas serológicas para los grupos con inoculación experimental. Los animales del grupo control resultaron negativos durante todo el desarrollo del experimento. Se observó una respuesta de seroconversión a partir del tercer dPI, resultando positivos todos los animales de los grupos intravenoso y de mucosas a los 21 y 28 días. Hubo una disminución de la seropositividad a los $56 \mathrm{y}$ $80 \mathrm{dPI}$ para los carneros de la vía mucosas y endovenosa (66 y 50\% de seropositividad, respectivamente). Al final del experimento (189 dPI), el 50\% de los animales de los grupos desafiados se presentaron como positivos o sospechosos a la prueba de ELISA.
El Cuadro 1 muestra las diferencias en la comparación de MIF a los 120 dPI entre el grupo control y el grupo vía endovenosa para CD4, encontrándose valores de $\mathrm{p}=0.03$, con rangos de 509.4 para el grupo control y de 604.7 para el endovenoso. Lo mismo ocurrió en los carneros del grupo control (1027.4) y el de vía endovenosa (499.6) para CD8 $(\mathrm{p}<0.05)$. Por otro lado, no se encontraron diferencias por efecto de grupo sobre las poblaciones de linfocitos CD4 ni CD8 a los 150 días.

En cuanto a las MIF a los 189 días posinfección para CD4 y CD8, se encontraron diferencias entre los carneros del grupo control y el endovenoso $(p<0.05)$, no así para el grupo control y el mucosal. Así mismo, se encontraron diferencias entre los grupos mucosal y endovenoso para CD4 a los 189 días pos-infección $(\mathrm{p}<0.05)$, en tanto que no se encontró diferencia significativa para CD8 entre los grupos en ese periodo.

Se encontraron diferencias en las MIF entre tiempos de infección a los 120,150 y 189 dPI en el grupo control $(p<0.05)$ en el caso de CD4, pero en CD8 solo se observaron diferencias significativas $(\mathrm{p}<0.05)$ entre los días 120 y 189 dPI (Cuadro 2). Por otro 


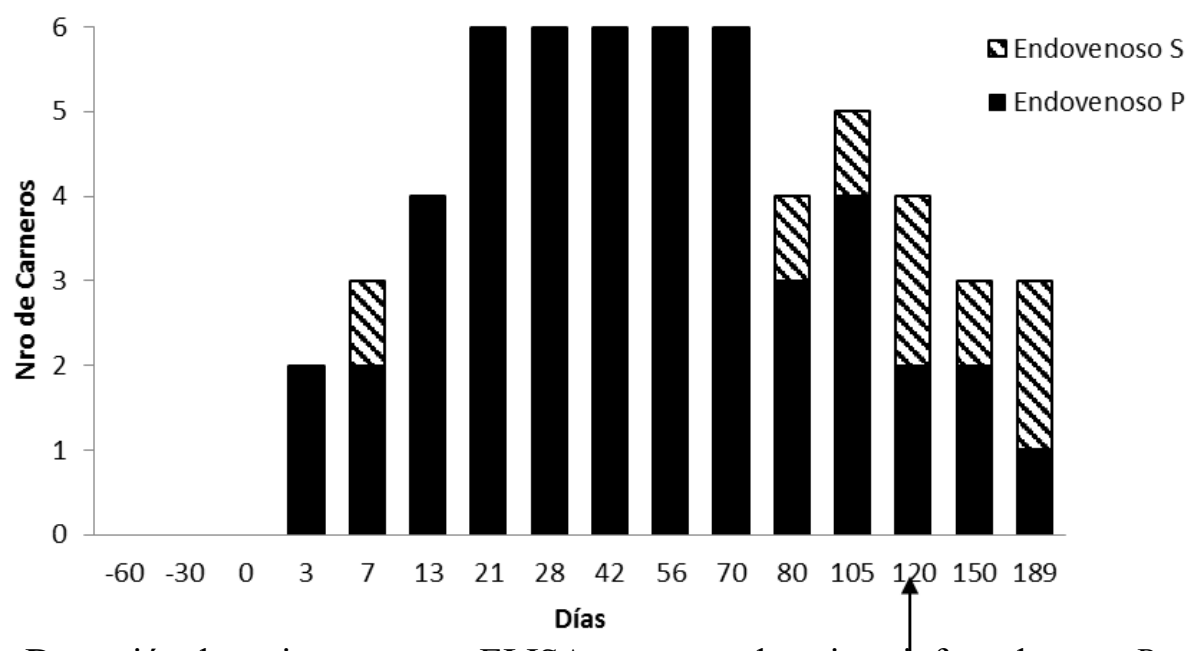

Figura 2. Detección de anticuerpos por ELISA en sueros de ovinos infectados con B. ovis por vía endovenosa con $1.0 \mathrm{ml}$ de inóculo con una concentración de $3.9 \times 10^{9}$ UFC. S: sospechoso; P: positivo. La flecha indica el momento en que se comenzó a realizar las lecturas de poblaciones linfocitarias (día 120 pos-infección)

Cuadro 1. Comparación de las Medias de Intensidad de Fluorescencia (MIF) de las poblaciones linfocitarias CD4 y CD8 entre grupos según el tiempo de infección por Brucella ovis

\begin{tabular}{lcccc}
\hline \multicolumn{2}{l}{ Días pos-infección } & Grupo control & Vía mucosas & Vía endovenosa \\
\hline 120 & & & & \\
& CD4 & $509.4^{\mathrm{a}}$ & $567.6^{\mathrm{ab}}$ & $604.7^{\mathrm{b}}$ \\
& CD8 & $1027.4^{\mathrm{a}}$ & $682.4^{\mathrm{ab}}$ & $499.6^{\mathrm{b}}$ \\
& & & & \\
& $\mathrm{CD} 4$ & $924.1^{\mathrm{a}}$ & $685.8^{\mathrm{a}}$ & $822.1^{\mathrm{a}}$ \\
& $\mathrm{CD} 8$ & $704.1^{\mathrm{a}}$ & $1063.7^{\mathrm{a}}$ & $724.2^{\mathrm{a}}$ \\
& & & & \\
& $\mathrm{CD} 4$ & $604.1^{\mathrm{a}}$ & $634.5^{\mathrm{a}}$ & $483.9^{\mathrm{b}}$ \\
& $\mathrm{CD} 8$ & $747.6^{\mathrm{a}}$ & $848.1^{\mathrm{a}}$ & $800.2^{\mathrm{a}}$ \\
\hline
\end{tabular}

$a, b, c$ Literales diferentes indican diferencias significativas $(p<0.05)$ entre grupos de infección

lado, se encontraron diferencias en el grupo mucosal, tanto para CD4 como para CD8, entre el grupo control y los grupos mucosal y endovenoso $(\mathrm{p}<0.05)$. En el grupo endovenoso se encontró diferencia significativa $(\mathrm{p}<0.05)$ por efecto de tiempo entre los grupos control y mucosal entre los $120 \mathrm{dPI}$ y los 150 y 189 dPI tanto para CD4 como para CD8 $(\mathrm{p}<0.05)$.
Las poblaciones linfocitarias $\mathrm{T} \gamma \delta$ también fueron marcadas con WC1 a los 120 dPI, habiéndose encontrado diferencia significativa entre el grupo control y el grupo mucosal $(\mathrm{p}<0.05)$, mientras que entre los carneros del grupo control y el endovenoso no existió diferencia por efecto de grupo a los 120 días pos-infección (Cuadro 3). 
Cuadro 2. Comparación de las Medias de Intensidad de Fluorescencia (MIF) entre tiempos de infección con $B$ ovis por grupos de las poblaciones linfocitarias CD4 y CD8

\begin{tabular}{clccc}
\hline \multirow{2}{*}{$\begin{array}{c}\text { Población } \\
\text { linfocitaria }\end{array}$} & \multirow{2}{*}{ Grupo } & \multicolumn{3}{c}{ Días pos-infección } \\
\cline { 2 - 5 } CD4 & Control & $236.5^{\mathrm{a}}$ & 150 & 189 \\
& Vía mucosas & $256.0^{\mathrm{a}}$ & $703.4^{\mathrm{b}}$ & $604.1^{\mathrm{c}}$ \\
& Vía endovenosa & $239.7^{\mathrm{a}}$ & $822.1^{\mathrm{b}}$ & $483.4^{\mathrm{a}}$ \\
& Control & $345.6^{\mathrm{a}}$ & $704.1^{\mathrm{ab}}$ & $747.6^{\mathrm{b}}$ \\
& VD8 & $268.8^{\mathrm{a}}$ & $1063.3^{\mathrm{b}}$ & $838.2^{\mathrm{b}}$ \\
& Vía mucosas & $204.0^{\mathrm{a}}$ & $724.2^{\mathrm{ab}}$ & $800.2^{\mathrm{b}}$ \\
\hline
\end{tabular}

$a, b, c$ Literales diferentes indican diferencias significativas $(p<0.05)$ entre tiempos de infección dentro de grupos

Cuadro 3. Comparación de las Medias de Intensidad de Fluorescencia (MIF) de las poblaciones linfocitarias $\mathrm{T} \gamma \delta \mathrm{WC} 1$ entre grupos a los 120 días de infección con $B$ ovis

\begin{tabular}{lc}
\hline Grupo & MIF \\
\hline Control & $1642.5^{\mathrm{a}}$ \\
Vía mucosas & $1251.8^{\mathrm{b}}$ \\
Vía endovenosa & $1103.0^{\mathrm{ab}}$ \\
\hline
\end{tabular}

a,b Superíndices diferentes entre grupos a los 120 días de infección indica diferencia estadística $(p<0.05)$

En la Figura 3 se muestra la inmunofenotipificación de linfocitos $\mathrm{T} \gamma \delta$ en carneros a los $120 \mathrm{dPI}$. Se observa la positividad de los CD8 en el cuadrante inferior derecho y la positividad de los linfocitos $\mathrm{T} \gamma \delta$ en el cuadrante izquierdo superior. Se destaca una similitud en el número de linfocitos $\mathrm{T} \gamma \delta$ para ambas vías de infección, de allí que no hubiera diferencias significativas a los $120 \mathrm{dPI}$ en las MIF de estas poblaciones entre las dos vías de inoculación.

La Figura 4 muestra los porcentajes de poblaciones linfocitarias que mostraron positividad a los marcadores CD4-Alexa flúor y CD8-Pe a los 120, 150 y 189 dPI. Se observa que $23 \%$ de la población linfocitaria a los $120 \mathrm{dPI}$ de la inoculación a través de las mucosas corresponde a linfocitos CD4, mientras que el 15\% corresponde a linfocitos CD8. En el caso de la inoculación por vía endovenosa, el $15 \%$ corresponde a linfocitos CD4 y el $11 \%$ a linfocitos CD8. A los 150 $\mathrm{dPI}$, los linfocitos CD4 del grupo inoculado a través de las mucosas representa el 19\% de la población linfocitaria y los linfocitos CD8 representan el 14\%; en tanto que el 14\% corresponde a CD4 y 7\% a linfocitos CD8 en el grupo inoculado vía endovenosa. A los 189 dPI, la población de linfocitos CD4 y CD8 de carneros infectados a través de las mucosas representan el 15 y $20 \%$, respectivamente, en tanto que las poblaciones CD4 y CD8 de los carneros infectados vía endovenosa representan 18 y $12 \%$, respectivamente.

En términos generales, se observa que con el transcurso del tiempo disminuyen las poblaciones de linfocitos CD4 de los carneros infectados por las mucosas; sin embargo, se observó un ligero aumento entre el día 120 al 189 en la población de CD8. Por otro lado, el comportamiento de los linfocitos CD4 y CD8 en carneros inoculados por vía endovenosa fue más variable. 


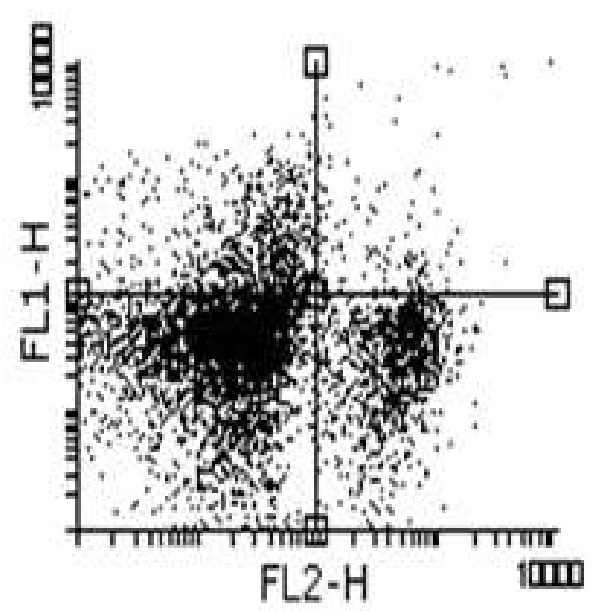

A

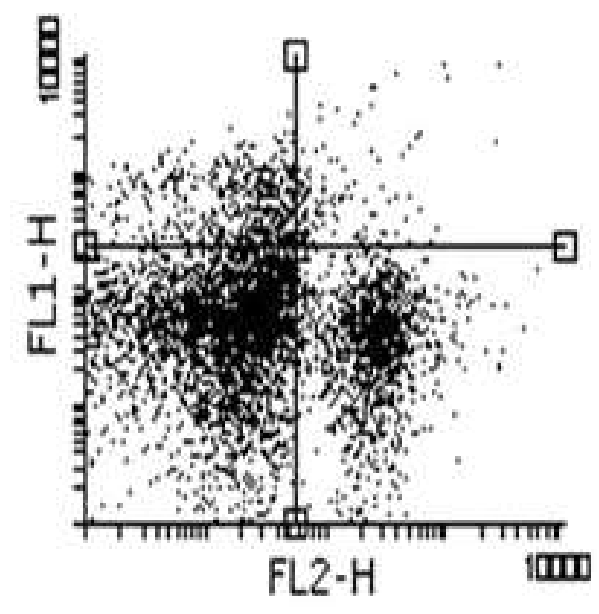

B

Figura 3. Histograma de lectura por citometría de flujo. Se observan linfocitos $T \gamma \delta$ (WC1) en carneros infectados con B. ovis por vía endovenosa (A) e infectados por vía mucosal (B). $120 \mathrm{dPI}$. En ambos casos, en el cuadrante superior derecho se observa la población de linfocitos $\mathrm{T} \gamma \delta$ (WC1), mientras que en el cuadrante inferior derecho se observan linfocitos CD8. (FL1: 1infocitos T $\gamma \delta$ (WC1) FITC; FL2: CD8 Pe)

\section{Discusión}

Las bacterias del género Brucella se multiplican dentro de los macrófagos del hospedador, lo que le permite evadir la respuesta inmune y complica el diagnóstico, ya que exhiben una gran variabilidad en la presentación de antígenos como en sus propiedades inmunomoduladoras (Baldwin y Goenka, 2006). Silva et al. (2013) trabajaron con IGDA encontrando respuesta positiva en todos los animales a los 30 días de la infección, tal y como ocurrió en el presente trabajo En forma similar, encontraron $50 \%$ de positividad en los carneros a los 168 días de la infección, siendo similar al resultado de 50\% a los $189 \mathrm{dPI}$ en ambos grupos inoculados de este estudio.

Las proteínas de membrana de las bacterias del género Brucella son antígenos potencialmente inmunogénicos que inducen repuesta inmune humoral y celular en ovinos (Cassataro et al., 2005) y tal como ocurre en otras infecciones causada por microor- ganismos intracelulares, tanto la participación de linfocitos CD4 y CD8 como la respuesta de anticuerpos median la inmunidad adquirida (Arranz-Solís et al., 2016).

La protección contra la brucelosis depende principalmente de la inmunidad mediada por células tales como los macrófagos, células dendríticas y linfocitos $\mathrm{T}$, tanto CD4 como CD8 (Cassataro et al., 2005). El IFN- $\gamma$ que es secretado por CD4 regula la actividad de los macrófagos, mientras que los linfocitos CD8 causan lisis en las células infectadas por las brucellas (Koch et al., 2012).

Al parecer, la participación de los linfocitos CD4 y CD8 puede variar dependiendo del transcurso de la infección y la modulación de la respuesta inmune acorde a la función de los linfocitos. Los CD4 demandan la repetición de la exposición de los antígenos para la proliferación y diferenciación de las células efectoras productoras de citoquinas (Bajénoff et al., 2002). Por otro lado, está demostrada la importancia de los linfocitos CD8 al producir un efecto citotóxico 


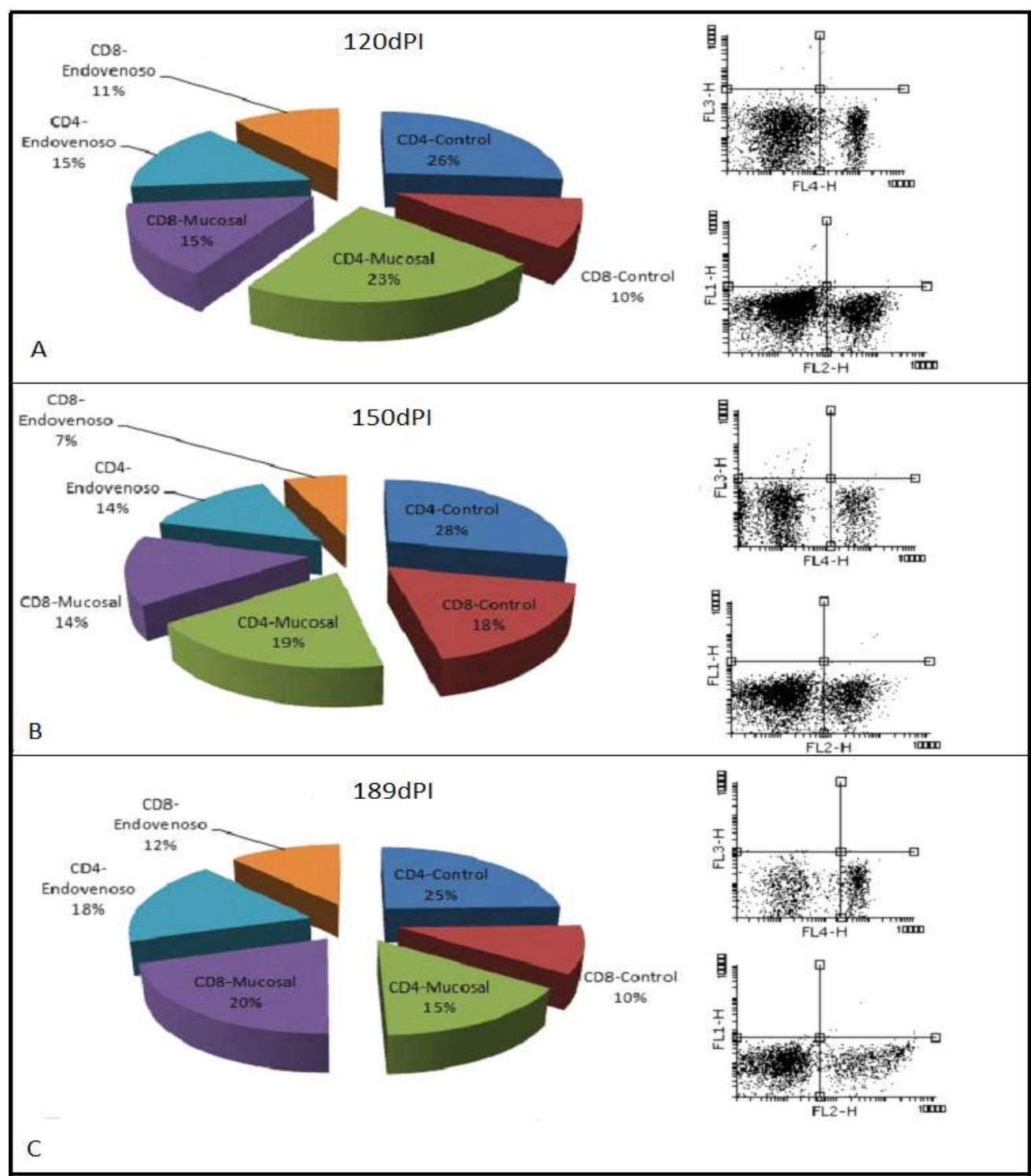

Figura 4. Poblaciones de linfocitos CD4 y CD8 que fueron marcados positivamente (CD4Alexa flúor; CD8-Pe) en tres grupos de carneros a los 120, 150 y 189 días posinfección. En el lado derecho de las gráficas se observan los histogramas de las lecturas por citometría de flujo de las poblaciones de linfocitos CD4 y CD8 de algunos carneros (FL2: CD8Pe; FL4: CD4-Alexa flúor)

en células infectadas por virus (Sánchez-Cordón et al., 2015) y otros microorganismos intracelulares (Arranz-Solís et al., 2016).

En este trabajo se encontraron diferencias en las MIF de las poblaciones linfocitarias CD8 del grupo control con los grupos inoculados en diferentes días pos-infección, lo que demuestra que la participación de CD4 y CD8 en los procesos crónicos de $B$. ovis puede ser determinante para el control de la infección (Díaz et al., 2013; Costa et al., 2016).

En el caso de infección por B. abortus, los linfocitos CD4 participan en el control y regulación del sistema inmune dependiendo 
de los perfiles de secreción de citoquinas, tanto proinflamatorios como antiinflamatorios (Martirosyan et al., 2013; Issuree et al., 2017). Con base a esto, se podría deducir que la fluctuación observada en este trabajo sea causada por los diferentes perfiles de citoquinas presentes en el tiempo que dura la infección; en tanto que la participación de $\mathrm{CD} 8$, al ser citotóxica, tendría menor variación.

En este trabajo se observaron diferencias en las MIF a los $120 \mathrm{dPI}$ en linfocitos $\mathrm{T} \gamma \delta$, lo cual demuestra su participación en esta infección, tal como se ha reportado en humanos para otras brucellas (van der Veken et al., 2005, Skendros et al., 2011). Estas poblaciones son importantes para el control de la infección con B. ovis en carneros donde el porcentaje de los linfocitos $\mathrm{T} \gamma \delta$ es mayor al 15\% (Skyberg et al., 2011). De acuerdo con lo reportado por otros autores, se deben realizar investigaciones adicionales para dilucidar el comportamiento de estos linfocitos.

\section{Conclusiones}

- Brucella ovis puede modular la respuesta inmune del hospedero, donde los linfocitos CD4 y CD8 son importantes para la defensa del hospedero ante este tipo de infección

- Las poblaciones de CD4 y CD8 pueden fluctuar a lo largo de la infección por $B$. ovis.

- La participación de los linfocitos $\mathrm{T} \gamma \delta$ podría ser un factor importante en el control de la infección causada por $B$. ovis.

\section{Agradecimientos}

Este trabajo de investigación fue financiado por el proyecto PROMEP/103.5/10/ 4368 y por el Consejo Nacional de Ciencia y Tecnología (CONACYT), bajo el Programa de Consolidación Institucional de Grupos de Investigación. Retención Ref: 09-01.117071. Los autores agradecen al Dr. Efren Diaz
Aparicio del CENID Microbiología México por el suministro de la cepa de Brucella ovis.

\section{Literatura Citada}

1. Acosta-Dibarrat J, Buendia-Jimenez A, Soriano-Vargas E, Montes De OcaJimenéz R, Tórtora-Perez J. 2014. Distribution of immune response cells in the pelvic urethra and the prepuce of rams. Pesqui Vet Bras 34: 270-276. doi: 10.1590/S0100-736X2014000300012

2. Acosta-Dibarrat J, Tórtora-Pérez J, Tenorio-Gutiérrez V, Soriano-Vargas E, Talavera-Rojas M, Cal-Pereyra L, Montes De Oca-Jiménez R, et al. 2016. Distribution of lymphocytes, immunoglobulin-containing cells, macrophages, and dendritic cells in the accessory sex glands of rams experimentally infected with Actinobacillus seminis. Pesqui Vet Bras 36: 363-372. doi: 10.1590/S0100-736X20160-00500-002

3. Albarrak SM, Waters WR, Satabel JR, Hostetter JM. 2017. $\mathrm{WC}^{+} \gamma \delta \mathrm{T}$ cells from cattle naturally infected with $\mathrm{Myco}$ bacterium avium subsp paratuberculosis respond differentially to stimulation with PPD-J. Vet Immunol Immunopathol 190: 57-64. doi: org/10.1016/j vetimm.2017.07.007

4. Allen JE, Maizels RM. 1997. Th1-Th2: reliable paradigm or dangerous dogma? Immunol Today 18: 387-392. doi: 10.1016/S0167-5699(97)01102-X

5. Arranz-Solís D, Benavides J, RegidorCarrillo J, Horcajo P, Castaño P, Ferreras MC, Jiménez-Pelayo L, et al. 2016. Systemic and local immune responses in sheep after Neospora caninum experimental infection at early, mid and late gestation. Vet Res 47: 2 . doi: 10.1186/s13567-015-0290-0

6. Bajénoff M, Wurtz O, Guerder S. 2002. Repeated antigen exposure is necessary for the differentation, but not the initial proliferation, of naive CD4(+) T cells. J Immunol 168: 1723-1729. doi: 10.4049/jimmunol.168.4.1723 
7. Baldwin CL, Goenka R. 2006. Host immune responses to the intracellular bacteria Brucella: does the bacteria instruct the host to facilitate chronic infection? Crit Rev Immunol 26: 407-442. doi: 10.1615/CritRevImmunol.v26.i5.30

8. Bessoles S, Ni M, Garcia-Jimenez S, Sanchez F, Lafont V. 2011. Role of NKG2D and its ligands in the antiinfectious activity of $\mathrm{V} \gamma 9 \mathrm{~V} \delta 2 \mathrm{~T}$ cells against intracellular bacteria. Eur J Immunol 41: 1619-1628. doi: 10.1002/ eji.201041230

9. Bowden RA, Cloeckaert A, Zygmunt MS, Dubray G. 1995. Outer membrane protein and rough lipopolysaccharide specific monoclonal antibodies protect mice against Brucella ovis. J Med Microbiol 43: 344-347. doi: 10.1099/ 00222615-43-5-344

10. Cannella AP, Tsolis RM, Liang L, Felgner PL, Saito M, Sette A, Gotuzzo $E$, et al. 2012. Antigen-specific acquired immunity in human brucellosis: implications for diagnosis, prognosis, and vaccine development. Front Cell Infect Mi 2: 1. doi: 10.3389/fcimb.2012.00001

11. Cassataro J, Velikovsky CA, de la Barrera S, Estein SM, Bruno L, Bowden $R$, Pasquevich KA, et al. 2005. A DNA vaccine coding for the Brucella outer membrane protein 31 confers protection against $B$ melitensis and $B$. ovis infection by eliciting a specific cytotoxic response. Infec Immun 73: 6537-6546. doi: 10.1128/IAI.73.10.6537-6546.2005

12. Cobbold SP, Adams E, Howie D, Waldmann H. 2018. CD4+ T cell fate decisions are stochastic, precede cell division, depend on GITR co-stimulation, and are associated with uropodium development. Front Immunol 9: 1381. doi: 10.3389/fimmu.2018.01381

13. Costa LF, Pessoa M, Guimarães L, Faria AK, Morão R, da Silva Mol J, Garcia JN, et al. 2016. Serologic and molecular evidence of Brucella ovis infection in ovine and caprine flocks in the State of Minas Gerais, Brazil. BMC
Res Notes 9: 190. doi: 10.1186/s13104016-1998-2

14. Cunnusamy K, Chen PW, Niederkorn JY. 2010. Paradigm shifts in the role of CD4+ T cells in keratoplasty. Discov Med 10: 452-461.

15. Díaz AG, Clausse M, Paolicchi FA, Fiorentino MA, Ghersi G, Zylberman V, Goldbaum FA, et al. 2013. Immune response and serum bactericidal activity against Brucella ovis elicited using a short immunization schedule with the polymeric antigen BLSOmp31 in rams. Vet Immunol Immunop 154: 36-41. doi: 10.1016/j.vetimm.2013.04.003

16. Dornand J, Gross A, Lafont V, Liautard J, Oliaro J, Liautard J-P. 2002. The innate immune response against Brucella in humans. Vet Microbiol 90: 383-394. doi: 10.1016/ S0378-1135(02)00223-7

17. Dudal S, Turriere C, Bessoles S, Fontes P, Sanchez F, Liautard J, Liautard JP, et al. 2016. Release of LL-37 by activated human Vgamma9Vdelta2 T cells: a microbicidal weapon against Brucella suis. J Immunol 177: 5533-5539. doi: 10.4049/jimmunol.177.8 .5533

18. Estein SM, Cheves PC, Fiorentino $M A$, Cassataro J, Paolicchi FA, Bowden RA. 2004. Immunogenicity of recombinant Omp31 from Brucella melitensis in rams and serum bactericidal activity against $B$. ovis. Vet Microbiol 102: 203-213. doi: 10.1016/j.vetmic.2004.05.004

19. Galindo RC, Muñoz PM, de Miguel MJ, Marin CM, Blasco JM, Gortazar C, Kocan KM, et al. 2009a. Characterization of possible correlates of protective response against Brucella ovis infection in rams immunized with the $B$. melitensis Rev 1 vaccine. Vaccine 27: 3039-3044.

20. Galindo RC, Muñoz PM, de Miguel MJ, Marin CM, Blasco JM, Gortazar C, Kocan KM, et al. 2009b. Differential expression of inflammatory and immune 
response genes in rams experimentally infected with a rough virulent strain of Brucella ovis. Vet Immunol Immunop 127: 295-303. doi: 10.1016/j.vetimm.2008.10.326

21. Goenka R, Parent MA, Elzer PH, Baldwin CL. 2011. B cell-deficient mice display markedly enhanced resistance to the intracellular bacterium Brucella abortus. J Infect Dis 203: 11361146. doi: 10.1093/infdis/jiq171

22. Hawkins RD, Larjo A, Tripathi SK, Wagner $U$, Luи Y, Lönnberg T, Raghav SK, et al. 2013. Global chromatin state analysis reveals lineagespecific enhancers during the initiation of human $\mathrm{T}$ helper 1 and T helper 2 cell polarization. Immunity 38: 1271-1284. doi: 10.1016/j.immuni.2013.05.011

23. Issuree PD, Ng CP, Littman DR. 2017. Heritable gene regulation in the CD4:CD8 T cell lineage choice. Front Immunol 8: 291. doi: 10.3389/fimmu.2017.00291

24. Jacques I, Cloeckaert A, Limet JN, Dubray G 1992. Protection conferred on mice by combinations of monoclonal antibodies directed against outer mem-brane proteins or smooth lipopoly-saccharide of Brucella. J Med Microbiol 37: 100-103. doi: 10.1099/00222615-37-2-100

25. Koch MA, Thomas KR, Perdue NR, Smigiel KS, Srivastava S, Campbell DJ. 2012. T-bet(+) Treg cells undergo abortive Th1 cell differentiation due to impaired expression of IL-12 receptor B2. Immunity 37: 501-510. doi: 10.1016/ j.immuni.2012.05.031

26. Littell RC. Henry PR, Ammerman CB. 1998. Statistical analysis of repeated measures data using SAS procedures. J Anim Sci 76:1216-1231.

27. Martirosyan A, Von Bargen K, ArceGorvel $V$, Zhao W, Hanniffy $S$, Bonnardel J, Méresse S, et al. 2013. In vivo identification and characterization of $\mathrm{CD} 4 \mathrm{z}$ cytotoxic $\mathrm{T}$ cells induced by virulent Brucella abortus infection. Plos One 8: e82508. doi: 10.1371/ journal.pone. 0082508
28. Miles AA, Misra SS, Irwin JO. 1938. The estimation of the bactericidal power of the blood. J Hyg 38: 732-749.

29. Moustacas VS, Silva TM, Costa LF, Xavier MN, Carvalho CA, Costa ÉA, Paixão TA, et al. 2013. Species-specific multiplex PCR for the diagnosis of Brucella ovis, Actinobacillus seminis, and Histophilus somni infection in rams. BMC Vet Res 9: 51. doi: 10.1186/17466148-9-51

30. Mucida D, Cheroutre H. 2010. The many face-lifts of CD4 T helper cells. Adv Immunol 107: 139-152, doi: 10.1016/ B978-0-12-381300-8.00005-8

31. Navarro JA, Caro MR, Seva J, Rosillo MC, Gomez MA, Gallego MC. 1996. Study of lymphocyte subpopulations in peripheral blood and secondary lymphoid organs in the goat using monoclonal antibodies to surface markers of bovine lymphocytes. Vet Immunol Immunopathol 51: 147-156. doi: 10.1016/01652427(95)05497-9

32. Ni M, Martire D, Scotet E, Bonneville M, Sanchez F, Lafont V. 2012. Full restoration of Brucella-infected dendritic cell functionality through $\mathrm{V} \gamma 9 \mathrm{~V} \delta 2 \mathrm{~T}$ helper type 1 crosstalk. Plos One 7: e43613. doi: 10.1371/journal.pone.0043613

33. Plattner BL, Hostetter JM. 2011. Comparative gamma delta $\mathrm{T}$ cell immunology: a focus on mycobacterial disease in cattle. Vet Med Int 2011: 214384. doi: $10.4061 / 2011 / 214384$

34. Praud A, Champion JL, Corde Y, Drapeau A, Meyer L, Garin-Bastuji B. 2012. Assessment of the diagnostic sensitivity and specificity of an indirect ELISA kit for the diagnosis of Brucella ovis infection in rams. BMC Vet Res 9: 68. doi: 10.1186/1746-6148-8-68

35. Ridler AL, Smith SL, West DM. 2014. Seroconversion and semen shedding in rams experimentally infected with Brucella ovis. New Zeal Vet J 62: 4750. doi: 10.1080/00480169.2013.836697 
36. Sánchez-Cordón PJ, Pérez de Diego AC, Gómez-Villamandos JC, SánchezVizcaíno JM, Pleguezuelos FJ, Garfía B, et al. 2015. Comparative analysis of cellular immune reponses and cytokine levels in sheep experimentally infected with bluetongue virus serotype 1 and 8 . Vet Microbiol 177: 95-105. doi:org/ 10.1016/vetmic.2015.02.022

37. Silva AP, Macêdo AA, Costa LF, Turchetti AP, Bull V, Pessoa MS, Araújo MS, et al. 2013. Brucella ovis lacking a species-specific putative ATPbinding cassette transporter is attenuated but immunogenic in rams. Vet Microbiol 167: 546-553. doi: 10.1016/j.vetmic.2013.-09.003

38. Skendros P, Pappas G, Boura P. 2011. Cell-mediated immunity in human brucellosis. Microbes Infect 13: 134-142. doi: 10.1016/j.micinf.2010.10.015

39. Skyberg JA, Thornburg T, Rollins M, Huarte E, Jutila MA, Pascual DW. 2011. Murine and bovine $\gamma \delta \mathrm{T}$ cells enhance innate immunity against Brucella abortus infections. Plos One 6: e21978. doi: 10.1371/journal.-pone.0021978
40. van der Veken LT, Hoogeboom M, de Paus RA, Willemze R, Falkenburg JH, Heemskerk MH. 2005. HLA class II restricted T-cell receptor gene transfer generates $\mathrm{CD} 4+\mathrm{T}$ cells with helper activity as well as cytotoxic capacity. Gene Ther 12: 1686-1695. doi: 10.1038/ sj.gt.3302586

41. Vitry MA, De Trez C, Goriely $S$, Dumoutier L, Akira S, Ryffel B, Carlier Y, et al. 2012. Crucial role of gamma interferon-producing CD4+ Th1 cells but dispensable function of $\mathrm{CD} 8+$ $\mathrm{T}$ cell, B cell, Th2, and Th17 responses in the control of Brucella melitensis infection in mice. Infect Immun 80: 42714280. doi: 10.1128/IAI.00761-12

42. Xavier MN, Silva TM, Costa EA, Paixão TA, Moustacas VS, Carvalho CA, Sant'Anna FM et al. 2010. Development and evaluation of a speciesspecific PCR assay for the detection of Brucella ovis infection in rams. Vet Microbiol 145: 158-164. doi: 10.1016/ j.vetmic.2010.02.037 